\title{
Systemic abnormalities of psoriatic patients: a retrospective study
}

This article was published in the following Dove Press journal:

Clinical, Cosmetic and Investigational Dermatology

10 November 2016

Number of times this article has been viewed

\section{$\operatorname{Li~Yin~}^{1, *}$ \\ Jia-Li Xu',* \\ Ying-Ying $\mathrm{Hu}^{\prime}$ \\ Andrew Johnston ${ }^{3}$ \\ Zhi-Qiang Yin'}

'Department of Dermatology,

${ }^{2}$ Department of Oncology, The

First Affiliated Hospital of Nanjing

Medical University, Nanjing, People's

Republic of China; ${ }^{3}$ Department of

Dermatology, University of Michigan,

Ann Arbor, MI, USA

*These authors contributed equally to this work

Correspondence: Zhi-Qiang Yin Department of Dermatology, The First Affiliated Hospital of Nanjing Medical University, 300 Guangzhou Road, Nanjing, Jiangsu 210029, People's Republic of China Email yzq2802@sina.com

Andrew Johnston

Department of Dermatology, University of Michigan, 6427 Medical Sciences

Building I, I30 I East Catherine Street,

Ann Arbor, MI 48I09, USA

Email andjoh@med.umich.edu
Background: Psoriasis is a chronic immune-mediated inflammatory disease related to the metabolic syndrome, cardiovascular disease, and other comorbidities. However, so far there has been no specific research concerning systemic abnormalities in psoriatic patients.

Objective: A retrospective study was conducted focusing on the detailed systemic abnormalities in psoriatic patients.

Methods: Psoriatic inpatients data was collected from July 2009 to September 2015. The inclusion criteria were first-time hospitalization and without administration of systemic drug therapy or exposure to phototherapy for psoriasis for at least 1 month. Detailed systemic indexes were mainly evaluated.

Results: The abnormality rates of blood routine examination, urine examination, blood biochemical examination and chest X-ray of 43 psoriatic patients were significantly higher than those of 44 non-psoriasis controls, and psoriasis patients significantly had higher absolute values of leukocytes and neutrophils, and significantly lower values of lymphocytes. Compared with psoriasis vulgaris, erythrodermic psoriasis had significantly higher abnormality rates of blood biochemical examination and serum electrolyte analysis. Erythrodermic psoriasis had significantly higher absolute values of blood leukocytes, neutrophils, and lower serum calcium compared with those of psoriasis vulgaris. The neutrophil-to-lymphocyte ratio of controls was significantly lower than that of psoriatic patients, and neutrophil-to-lymphocyte ratio of erythrodermic psoriasis was significantly higher in comparison with psoriasis vulgaris.

Conclusion: This study is the first report in relation to a detailed assessment of systemic abnormalities in psoriatic patients prior to onset of systemic treatment. The systemic condition of psoriatic patients should be observed by clinicians before systemic therapy.

Keywords: psoriasis, skin, inflammation, treatment

\section{Introduction}

Psoriasis is a chronic immune-mediated inflammatory disease which primarily affects the skin and joints (psoriatic arthritis [PsA]), and has been related to metabolic syndrome and some manifestations of cardiovascular disease. Additionally, other comorbidities including Crohn's disease, autoimmune disease, liver disease, malignancy, chronic obstructive pulmonary disease, sleep apnea, uveitis, and depression are associated with psoriasis. ${ }^{1,2}$ As such, psoriasis is generally considered as a systemic inflammatory disease.

There are many studies measuring different serum cytokines and growth factors in psoriasis - some of them correlating with disease severity, such as tumor necrosis factor (TNF)- $\alpha$, interferon- $\gamma$, interleukin (IL)-12, IL-17A, and IL-18. ${ }^{3,4}$ Many of these studies 
were looking for biomarkers of disease - for example, TNF- $\alpha$, IL-6, IL-8, IL-17A, soluble TNF receptors-1, and lipocalin 2. 5,6 Meziane et al reported that abdominal obesity and dyslipidemia were metabolic factors significantly related to psoriasis, and hypertension, diabetes, increased levels of triglycerides, and high-density lipoprotein cholesterol were associated with the severity of psoriasis in Moroccan patients with psoriasis. ${ }^{7}$ However, so far there has been no specific research into detailed systemic abnormalities in psoriatic patients. Herein, we conducted a 6-year retrospective study focusing on the patients of first-time hospitalization with psoriasis.

\section{Patients and methods}

\section{Ethics statement}

This retrospective study was approved by Institutional Review Board of First Affiliated Hospital of Nanjing Medical University. Written informed consent was obtained from all patients, and the patient records were anonymized and de-identified prior to analysis.

\section{Study population}

Psoriatic inpatient data were collected in the Department of Dermatology of The First Affiliated Hospital of Nanjing Medical University in China from July 2009 to September 2015. The inclusion criteria were first-time hospitalization and without administration of systemic drug therapy (including systemic immunosuppressant, retinoic acid, and biologics or specific antibiotics) or exposure to phototherapy for psoriasis for at least 1 month (biologics should not have been given for at least 3 months).

Detailed systemic indices were evaluated including blood routine examination (BRE), urine examination (UE), blood biochemical examination (BBE, including liver function, renal function, serum lipids, and fasting blood glucose, etc), serum electrolyte analysis (SEA), chest X-ray (CXR), electrocardiogram (ECG), and abdominal ultrasonography (USG). Psoriatic patients were compared with non-psoriasis control subjects whom were randomly selected from the medical examination center in the same hospital.

\section{Statistics}

Abnormality rates of each group were calculated and statistical significance was calculated using chi-square test. All absolute counts are presented as mean \pm standard deviation, and statistical significance calculated using a two-tailed Student's $t$-test with subsequent Bonferroni correction, or Mann-Whitney $U$ test, as appropriate. Significance was defined as $P \leq 0.05$.

\section{Results}

From July 2009 to September 2015, 203 psoriatic inpatients were admitted to the Department of Dermatology of The First Affiliated Hospital of Nanjing Medical University, with 43 cases meeting the inclusion criteria including: 22 moderate-to-severe psoriasis vulgaris (PV) ([Psoriasis Area Severity Index] PASI >10), 15 erythrodermic psoriasis (EP), three generalized pustular psoriasis, and three PsA.

Seven of the 43 patients were female and all patients were $>18$ years old with an average age of 50 years. Eight of the 43 patients did not receive systemic treatment for psoriasis for 1 month, and the other 35 patients were not given systemic treatment within 3 months. The disease course of psoriatic patients was from 10 days to 50 years. The number of psoriatic patients with winter flares was 23 , and non-winter flares was 17 . We randomly chose 44 non-psoriasis control subjects from the medical examination center with a matching age and sex ratio. Any value that was not within the defined normal value range was defined as an "abnormality".

The comparison of detailed systemic abnormalities between psoriatic patients and non-psoriasis controls can be seen in Table 1 and Figure 1, which show that the abnormality rates of BRE, UE, BBE, and CXR of psoriatic patients were significantly higher than those of non-psoriasis controls. ( $P<0.001, P=0.021, P<0.001$, and $P<0.001$, respectively). In BRE, the incidence rates of leukocytosis, neutrophilia, hypolymphemia, and erythropenia (or hypochromasia) of psoriasis were significantly higher in comparison with controls ( $P=0.017, P<0.001, P<0.001$, and $P=0.032$, respectively). In UE, psoriatic patients had significantly higher incidence rate of leukocytosis compared with controls $(P=0.003)$. In BBE, the incidence rates of hypoalbuminemia, abnormal bilirubin, hyperalphalipoproteinemia, and high-density lipoprotein decrease in controls were all significantly lower than those of psoriatic patients $(P<0.001, P=0.032, P=0.004$, and $P=0.003$, respectively). Psoriatic patients had common serum electrolyte disturbances (46.51\%), especially hypocalcemia (27.91\%).

Table 2 and Figure 2A show that PV had high incidence rates of abnormal BRE (59.09\%), BBE (95.45\%), and CXR $(63.16 \%)$. The corresponding values of EP were $93.33 \%$, $93.33 \%$, and $71.43 \%$, respectively, and EP also had a high incidence rate of serum electrolyte disturbances (73.33\%). Compared with PV, EP had significantly higher abnormality rates of BRE and SEA ( $P=0.028$ and $P=0.045$, respectively).

We also compared psoriasis with winter flares with nonwinter flares. Table 3 and Figure 2B show that the abnormality rates of BRE, UE, BBE, SEA, CXR, ECG, and abdominal 
Table I The comparison of detailed systemic abnormalities between psoriatic patients and non-psoriasis controls

\begin{tabular}{|c|c|c|c|c|}
\hline Abnormality & Psoriasis $(n=43)$ & Controls $(n=44)$ & $\chi^{2}$ values & $P$-values \\
\hline Blood routine examination & $32(74.42)$ & $12(27.27)$ & 19.338 & $<0.001$ \\
\hline Leukocytosis & II (25.58) & $3(6.8 \mathrm{I})$ & 5.67 & 0.017 \\
\hline Neutrophilia & $15(34.88)$ & I (2.27) & $|5.4|$ & $<0.001$ \\
\hline Eosinophilia & $7(16.28)$ & I (2.27) & 3.57 & 0.059 \\
\hline Hypolymphemia & $17(39.53)$ & I (2.27) & 18.402 & $<0.001$ \\
\hline Monocytosis & $7(16.28)$ & $3(6.8 \mathrm{I})$ & 1.096 & 0.295 \\
\hline Erythropenia or hypochromasia & $8(18.6)$ & I (2.27) & 4.617 & 0.032 \\
\hline Thrombocytosis & $8(18.6)$ & $2(4.55)$ & 2.956 & 0.086 \\
\hline Thrombocytopenia & $3(6.98)$ & $0(0)$ & 1.429 & 0.232 \\
\hline Urine examination & $15(34.88)$ & $6(13.64)$ & 5.362 & 0.021 \\
\hline Leukocytosis & $10(23.26)$ & I (2.27) & 8.669 & 0.003 \\
\hline Erythremia & $4(9.3)$ & $3(6.82)$ & 0.001 & 0.975 \\
\hline Urine protein & $6(13.95)$ & $4(9.09)$ & 0.14 & 0.708 \\
\hline Hyperglycosuria & $5(11.63)$ & I (2.27) & 1.686 & 0.194 \\
\hline Blood biochemical examination & $40(93.02)$ & $18(40.91)$ & 26.578 & $<0.001$ \\
\hline Hepatic dysfunction & II (25.58) & $6(13.64)$ & 1.974 & 0.16 \\
\hline Renal dysfunction & $2(4.65)$ & $0(0)$ & N/A & 0.241 \\
\hline Hyperuricaemia & $7(16.28)$ & $3(6.82)$ & 1.096 & 0.295 \\
\hline Hyperglycemia & $7(16.28)$ & $2(4.55)$ & 2.087 & 0.149 \\
\hline Hypoalbuminemia & $21(48.84)$ & I (2.27) & 24.958 & $<0.001$ \\
\hline Abnormal bilirubin & $8(18.6)$ & I (2.27) & 4.617 & 0.032 \\
\hline Hyperalphalipoproteinemia & $9(20.93)$ & $0(0)$ & 8.139 & 0.004 \\
\hline High-density lipoprotein decrease & $23(53.49)$ & $10(22.73)$ & $8.74 I$ & 0.003 \\
\hline Serum electrolyte analysis & $20(46.5 I)$ & N/A & $\mathrm{N} / \mathrm{A}$ & N/A \\
\hline Hypokalemia & $5(11.63)$ & $\mathrm{N} / \mathrm{A}$ & $\mathrm{N} / \mathrm{A}$ & $N / A$ \\
\hline Hypocalcemia & $12(27.91)$ & $\mathrm{N} / \mathrm{A}$ & $\mathrm{N} / \mathrm{A}$ & $\mathrm{N} / \mathrm{A}$ \\
\hline Hyposodemia & I (2.34) & $\mathrm{N} / \mathrm{A}$ & $\mathrm{N} / \mathrm{A}$ & N/A \\
\hline Hypophosphatemia & $2(4.65)$ & N/A & $\mathrm{N} / \mathrm{A}$ & N/A \\
\hline Chest X-ray & $25(64.1, n=39)$ & $4(9.09)$ & 27.524 & $<0.001$ \\
\hline Electrocardiogram & I5 (39.47, n=38) & $13(29.55)$ & 0.894 & 0.344 \\
\hline Abdominal ultrasonography & $18(62.07, n=29)$ & $26(59.09)$ & 0.065 & 0.799 \\
\hline
\end{tabular}

Note: Data presented as n (\%).

Abbreviation: N/A, not applicable.

USG of psoriasis with winter flares had no significant difference compared with those of non-winter flares $(P>0.05)$.

Figure 3 shows the scatter plots of absolute values of blood leukocytes, lymphocytes, neutrophils, fasting blood glucose, and serum calcium. Figure 3A shows the comparison between psoriasis and non-psoriasis controls; the former had significantly higher absolute values for leukocytes and neutrophils ( $P=0.001$ and $P<0.001$, respectively), and significantly lower values for lymphocytes $(P<0.001)$. Fasting blood glucose levels of psoriasis patients were not significantly different to those of controls $(Z=-0.658, P=0.511)$. Figure 3B shows EP had significantly higher absolute values of blood leukocytes, neutrophils, and lower serum calcium compared with those of $\mathrm{PV}(P=0.01, P=0.022$, and $P=0.03$, respectively). EP and $\mathrm{PV}$ had no significant differences in absolute values for blood lymphocytes $(P=0.502)$ and blood glucose $(Z=-0.557, P=0.578)$. The neutrophil-to-lymphocyte ratio (NLR) in the controls was significantly lower than that of psoriatic patients $(P<0.001)$, and NLR of EP was significantly higher in comparison with PV $(P=0.033)$.

\section{Discussion}

Growing evidence from the last 10 years suggests that several systemic conditions like metabolic syndrome, cardiovascular disease, diabetes, psychological disorders, or inflammatory bowel disease are prevalent in psoriatic patients, and the link might be the chronic secretion of pro-inflammatory cytokines by immune cells and skin. ${ }^{8}$ Drug adverse reaction was deemed to be the cause of abnormalities in BRE and BBE of psoriatic patients in most cases. In this study, we eliminated the possible influence of systemic medications on psoriatic inpatients to evaluate the systemic abnormalities of psoriasis objectively and accurately. In our study, the most common comorbidity was metabolic disorder, according to examination results.

The psoriatic patients commonly had elevated peripheral blood leukocytes and neutrophils, which was shown in our 

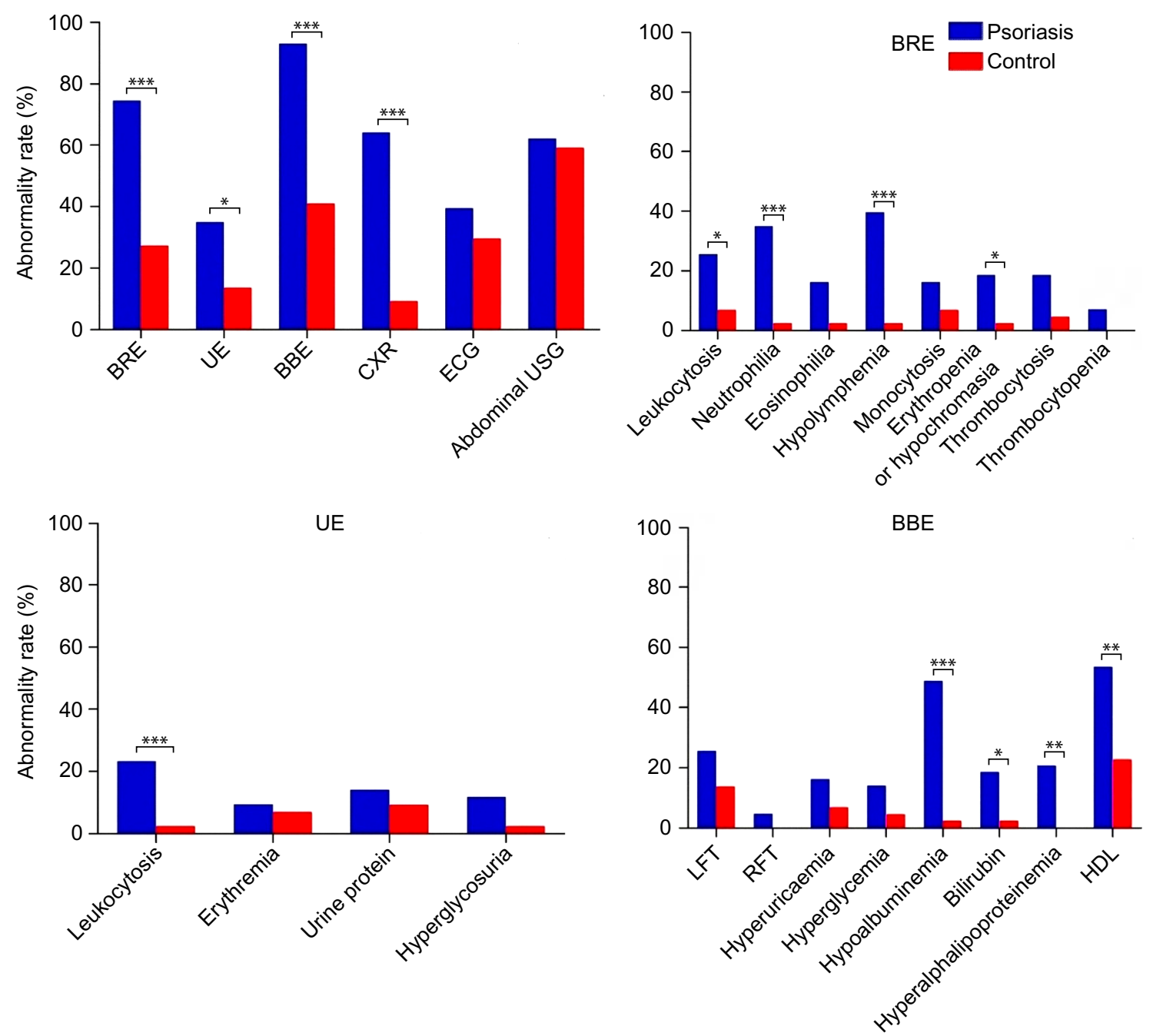

Figure I The comparison of abnormality rate between psoriatic patients and non-psoriasis controls.

Notes: Statistical significance indicated as $* P \leq 0.05, * * P \leq 0.01$, $* * * P \leq 0.001$.

Abbreviations: BRE, blood routine examination; UE, urine examination; BBE, blood biochemical examination; CXR, chest X-ray; ECG, electrocardiogram; USG, ultrasonography; LFT, liver function tests; RFT, renal function tests; HDL, high-density lipoprotein.

Table 2 The systemic abnormalities of PV, EP, GPP, and PsA

\begin{tabular}{|c|c|c|c|c|c|c|}
\hline Abnormality & PV $(n=22)$ & $\operatorname{EP}(n=15)$ & $X^{2}$ values & $P$-values & GPP $(n=3)$ & $\operatorname{PsA}(n=3)$ \\
\hline Blood routine examination & $13(59.09)$ & $14(93.33)$ & $\mathrm{N} / \mathrm{A}$ & 0.028 & $3(100)$ & $2(66.67)$ \\
\hline Urine examination & $6(27.27)$ & $5(33.33)$ & $N / A$ & 0.728 & $3(100)$ & I (33.33) \\
\hline Blood biochemical examination & $21(95.45)$ & $14(93.33)$ & $\mathrm{N} / \mathrm{A}$ & I & $3(100)$ & $2(66.67)$ \\
\hline Serum electrolyte analysis & $8(36.36)$ & II (73.33) & $\mathrm{N} / \mathrm{A}$ & 0.045 & $0(0)$ & I (33.33) \\
\hline Chest X-ray & $12(63.16, n=19)$ & $10(7 \mid .43, n=14)$ & $\mathrm{N} / \mathrm{A}$ & 0.719 & $2(66.67)$ & I (33.33) \\
\hline Electrocardiogram & $8(42.11, n=19)$ & $5(38.46, n=13)$ & $\mathrm{N} / \mathrm{A}$ & I & I (33.33) & I (33.33) \\
\hline Abdominal ultrasonography & II $(64.71, n=17)$ & $4(57.14, n=7)$ & $N / A$ & I & I (33.33) & $2(66.67)$ \\
\hline
\end{tabular}

Note: Data presented as $\mathrm{n}(\%)$.

Abbreviations: PV, psoriasis vulgaris; EP, erythrodermic psoriasis; GPP, generalized pustular psoriasis; PsA, psoriatic arthritis; N/A, not applicable.

study, and it also showed EP had higher absolute counts of leukocytes and neutrophils than that of moderate-to-severe PV. NLR has been used as a marker for systemic inflammation status, and was considered to be a measure of systemic inflammation in psoriasis. ${ }^{9}, 10$ NLR was also deemed to be potentially a predictor of subclinical atherosclerosis in patients with psoriasis. ${ }^{11}$ Our study showed that psoriasis had higher NLR compared with non-psoriasis controls, and EP had higher NLR in comparison with PV, in parallel, EP had higher disease severity (PASI) scores, indicating that EP had more intense systemic inflammation.

Anemia is not a commonly-reported comorbidity of psoriasis. However, our study found psoriatic patients had a higher incidence rate of peripheral blood erythropenia 

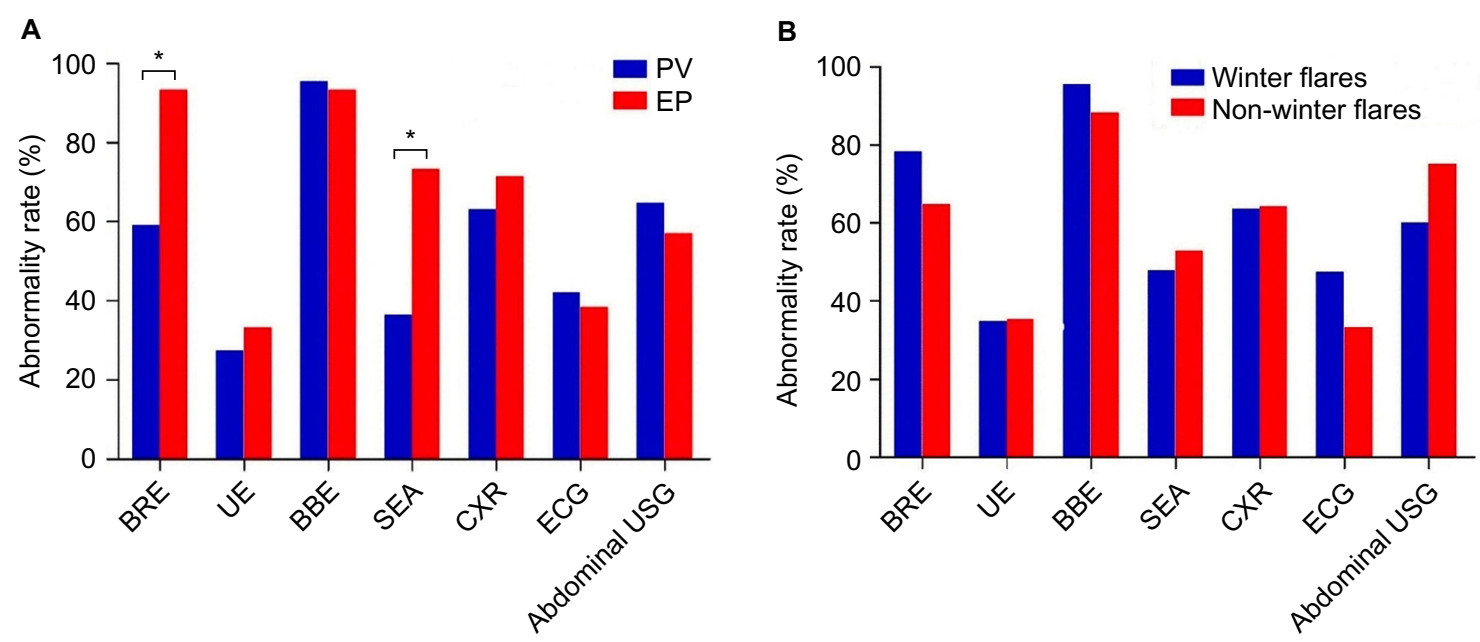

Figure 2 The comparison of abnormality rate between PV and EP $(\mathbf{A})$, and the comparison between psoriasis with winter flares and non-winter flares (B). Note: Statistical significance indicated as $* P \leq 0.05$.

Abbreviations: PV, psoriasis vulgaris; EP, erythrodermic psoriasis; BRE, blood routine examination; UE, urine examination; BBE, blood biochemical examination; SEA, serum electrolyte analysis; CXR, chest X-ray; ECG, electrocardiogram; USG, ultrasonography.

Table 3 The comparison of systemic abnormalities between psoriasis with winter flares and non-winter flares

\begin{tabular}{|c|c|c|c|c|}
\hline Abnormality & $\begin{array}{l}\text { Winter } \\
\text { flares }(n=23)\end{array}$ & $\begin{array}{l}\text { Non-winter } \\
\text { flares }(n=I 7)\end{array}$ & $\begin{array}{l}X^{2} \\
\text { values }\end{array}$ & P-values \\
\hline $\begin{array}{l}\text { Blood routine } \\
\text { examination }\end{array}$ & I 8 (78.26) & I I (64.7I) & 0.349 & 0.555 \\
\hline Urine examination & $8(34.78)$ & $6(35.29)$ & 0.001 & 0.973 \\
\hline $\begin{array}{l}\text { Blood biochemical } \\
\text { examination }\end{array}$ & $22(95.65)$ & $15(88.24)$ & 0.075 & 0.785 \\
\hline $\begin{array}{l}\text { Serum electrolyte } \\
\text { analysis }\end{array}$ & II (47.83) & $9(52.94)$ & 0.102 & 0.749 \\
\hline Chest X-ray & $14(63.64, n=22)$ & $9(64.29, n=14)$ & $\mathrm{N} / \mathrm{A}$ & I \\
\hline Electrocardiogram & $9(47.37, n=19)$ & $5(33.33, n=15)$ & N/A & 0.495 \\
\hline $\begin{array}{l}\text { Abdominal } \\
\text { ultrasonography }\end{array}$ & $9(60, n=15)$ & $9(75, n=12)$ & N/A & 0.683 \\
\hline
\end{tabular}

Note: Data presented as $n$ (\%).

Abbreviation: N/A, not applicable.

or hypochromasia compared with non-psoriasis controls. Harada et al reported long-term adalimumab treatment might cause autoimmune hemolytic anemia which resolved after replacing the adalimumab treatment with prednisolone therapy. ${ }^{12}$ Drug side-effects might be a possible reason for erythropenia or hypochromasia of psoriasis, although we tried to lessen drug interference in our study design, nevertheless, we should take note of the possible abnormality in peripheral blood erythrocytes and hemoglobin of psoriatic patients.

Clinicians seldom pay attention to the urine of psoriatic patients, except to the presence of urine protein. Urinary proteome analysis could identify potentially predictive biomarkers for methotrexate-induced hepatic fibrosis in psoriasis with high cumulative dose of methotrexate. ${ }^{13}$ Interestingly, our study found psoriasis had more abnormalities in UE in comparison with non-psoriasis controls, and the main manifestation was urine leukocytosis, which suggested that psoriatic patients were prone to urinary infections, particularly as psoriatic patients treated with immunosuppressants or biological agents such as anti-TNF- $\alpha$ agents are at an increased risk of infections.

In BBE, 40 of 43 psoriatic patients had abnormalities including hypoalbuminemia, abnormal bilirubin, hyperalphalipoproteinemia, and high-density lipoprotein decrease, which were related to metabolic disturbance and had significantly higher incidence rates than non-psoriasis controls. Psoriasis has been reported to be associated with raised serum uric acid levels and increased risk of developing type 2 diabetes. ${ }^{14,15}$ However, in our study the incidence rate of hyperuricaemia and hyperglycemia was not significantly different between psoriasis patients and controls, which might be due to the small sample size. Fasting blood glucose levels of PV also had no marked difference in comparison with EP. Interestingly, moderate-to-severe PV and EP both had high abnormality rates in BBE but there were no significant differences, which indicated that moderate-to-severe PV might have similar severe systemic inflammation to EP.

Almost half (46.51\%) of psoriatic patients had serum electrolyte disturbances, and the main manifestation was hypocalcemia. Hypocalcemia is a risk factor for psoriasis. ${ }^{16}$ A recent study reported that serum calcium levels could predict the efficacy of methotrexate in the treatment of severe plaque psoriasis, and better improvements were observed in psoriatic patients with higher serum calcium. ${ }^{17}$ In our study, 

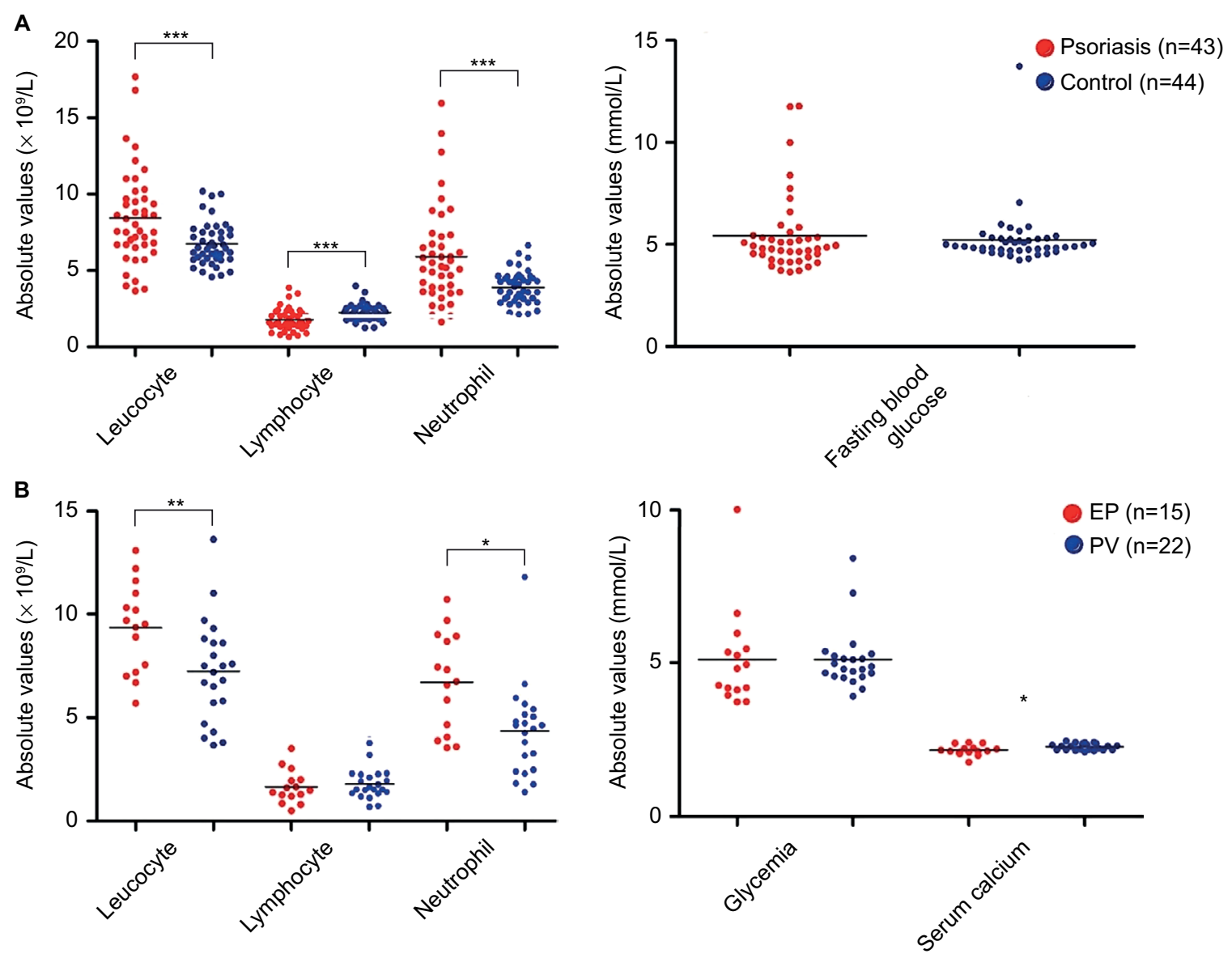

Figure 3 Scatter plots of absolute values of peripheral blood leukocyte, lymphocyte, neutrophil, fasting blood glucose, and serum calcium.

Notes: $(\mathbf{A})$ The comparison between psoriasis and non-psoriasis controls. (B) The comparison between EP and PV. Statistical significance indicated as $* P \leq 0.05$, $* * P \leq 0.01$, $* * * P \leq 0.001$.

Abbreviations: PV, psoriasis vulgaris; EP, erythrodermic psoriasis.

comparing moderate-to-severe PV with EP, the latter had higher disease severity (PASI) scores but significantly lower serum calcium levels, which may be a useful predictor of EP treatment outcomes.

Abnormal CXR signs could be seen in $64.1 \%$ of psoriatic patients which was mainly expressed in increased lung markings, whereas the abnormality rate of CXR in non-psoriasis controls was $9.09 \%$. Psoriatic patients were at a greater risk of developing chronic obstructive pulmonary disease than the general population and that the association between of psoriasis and with chronic obstructive pulmonary disease was stronger among patients with severe psoriasis. ${ }^{18}$ As a mark of early lung injury, increase of lung markings should be paid close attention to.

Our study found the main abnormality of ECG in psoriatic patients was sinus irregularity, which had no significant difference compared with non-psoriasis controls. However, a recent study with a large sample size reported that patients with psoriasis were at higher risk of developing arrhythmia, particularly for those with PsA, independent of traditional cardiovascular risk factors. ${ }^{19}$ Trial conduction of sinus impulses was impaired in patients with $\mathrm{PV}$, which was more prominent in patients with severe disease, and physicians caring for patients with PV should screen them for atrial fibrillation development. ${ }^{20}$ In our study, USG abnormality in psoriatic patients was mainly fatty liver; however, the incidence rate also had no marked difference compared with the controls. However, in another study, Gisondi et al reported that prevalence of nonalcoholic fatty liver disease was greater in patients with psoriasis than in controls, and fibrosis score of nonalcoholic fatty liver disease was also higher in psoriasis, suggesting a higher risk of liver fibrosis in those with psoriasis. ${ }^{21}$

The majority of psoriatic patients grow worse in the cold winter but improve in the summer months, whereas other patients have no such distinguishing features or illness is aggravated in summer. ${ }^{22}$ Various examinations in psoriasis with winter flares seemed to have no significant difference in 
comparison with those of non-winter flares; however, we considered differences might appear with a sample size increase.

\section{Limitations}

The major limitations of this study are its retrospective and inpatient nature. Patients who are admitted are different from patients who are seen in the outpatient setting: inpatients generally have a much more severe illness than outpatients. Another limitation is that this was a single center research. However, fortunately, the electronic data of patients and nonpsoriasis controls were quite comprehensive and comparable; moreover, our rigorous inclusion criteria rendered this study a dependable result.

\section{Conclusion}

To our knowledge, our study is the first report in relation to the detailed assessment about systemic abnormalities of psoriatic patients prior to systemic treatment. The systemic condition of psoriatic patients should be observed by clinicians before systemic therapy, and systemic abnormalities of different types of psoriasis should be taken care of during patient care.

\section{Acknowledgments}

This work was supported by the National Natural Science Foundation of China (81301387) and the Priority Academic Program Development of Jiangsu Higher Education Institutions (JX10231801).

\section{Disclosure}

The authors report no conflicts of interest in this work.

\section{References}

1. Duffin KC. Identifying and managing complications and comorbidities in patients with psoriasis. Semin Cutan Med Surg. 2015;34(2 Suppl):S30-33.

2. Kim N, Thrash B, Menter A. Comorbidities in psoriasis patients. Semin Cutan Med Surg. 2010;29(1):10-15.

3. Baliwag J, Barnes DH, Johnston A. Cytokines in psoriasis. Cytokine. 2015;73(2):342-350.

4. Takahashi H, Tsuji H, Hashimoto Y, Ishida-Yamamoto A, Iizuka H. Serum cytokines and growth factor levels in Japanese patients with psoriasis. Clin Exp Dermatol. 2010;35(6):645-649.
5. Fotiadou C, Lazaridou E, Sotiriou E, Gerou S, Kyrgidis A, Vakirlis E, Ioannides D. IL-17A, IL-22, and IL-23 as markers of psoriasis activity: a cross-sectional, hospital-based study. J Cutan Med Surg. 2015;19(6):555-560.

6. Ataseven A, Kesli R, Kurtipek GS, Ozturk P. Assessment of lipocalin 2 , clusterin, soluble tumor necrosis factor receptor-1, interleukin-6, homocysteine, and uric acid levels in patients with psoriasis. Dis Markers. 2014;2014:541709.

7. Meziane M, Kelati A, Najdi A, Berraho A, Nejjari C, Mernissi FZ. Metabolic syndrome in Moroccan patients with psoriasis. Int J Dermatol. 2016;55(4):396-400.

8. Voiculescu VM, Lupu M, Papagheorghe L, Giurcaneanu C, Micu E. Psoriasis and metabolic syndrome--scientific evidence and therapeutic implications. J Med Life. 2014;7(4):468-471.

9. Sen BB, Rifaioglu EN, Ekiz O, Inan MU, Sen T, Sen N. Neutrophil to lymphocyte ratio as a measure of systemic inflammation in psoriasis. Cutan Ocul Toxicol. 2014;33(3):223-227.

10. Ataseven A, Bilgin AU, Kurtipek GS. The importance of neutrophil lymphocyte ratio in patients with psoriasis. Mater Sociomed. 2014; 26(4):231-233.

11. Yurtdasş M, Yaylali YT, Kaya Y, Ozdemir M, Ozkan I, Aladă̆ N. Neutrophil-to-lymphocyte ratio may predict subclinical atherosclerosis in patients with psoriasis. Echocardiography. 2014;31(9):1095-1104.

12. Harada Y, Yamamoto H, Sato M, Kodaira M, Kono T. Autoimmune hemolytic anemia during adalimumab treatment for plaque psoriasis. Intern Med. 2015;54(9):1103-1104.

13. van Swelm RP, Laarakkers CM, Kooijmans-Otero M, de Jong EM, Masereeuw R, Russel FG. Biomarkers for methotrexate-induced liver injury: urinary protein profiling of psoriasis patients. Toxicol Lett. 2013;221(3):219-224.

14. Lai YC and Yew YW. Psoriasis and uric acid: a population-based crosssectional study. Clin Exp Dermatol. 2016;41(3):260-266.

15. Gyldenløve M, Vilsbøll T, Holst JJ, Zachariae C, Skov L, Knop FK. Disturbed postprandial glucose metabolism and gut hormone responses in non-diabetic patients with psoriasis. Br J Dermatol. Epub 2015 Mar 27.

16. Qadim HH, Goforoushan F, Nejad SB, Goldust M. Studying the calcium serum level in patients suffering from psoriasis. Pak J Biol Sci. 2013;16(6):291-294.

17. Zhai Z, Chen L, Yang H, et al. Can pretreatment serum calcium level predict the efficacy of methotrexate in the treatment of severe plaque psoriasis? J Am Acad Dermatol.2015;73(6):991-997.

18. Li X, Kong L, Li F, et al. Association between psoriasis and chronic obstructive pulmonary disease: a systematic review and meta-analysis. PLoS One. 2015;10(12):e0145221.

19. Chiu HY, Chang WL, Huang WF, Wen YW, Tsai YW, Tsai TF. Increased risk of arrhythmia in patients with psoriatic disease: a nationwide populationbased matched cohort study. J Am Acad Dermatol. 2015;73(3):429-438.

20. Bacaksiz A, Erdogan E, Tasal A, et al. Electrocardiographic P-wave characteristics in patients with psoriasis vulgaris. Ups J Med Sci. 2013;118(1):35-41.

21. Gisondi P, Barba E, Girolomoni G. Non-alcoholic fatty liver disease fibrosis score in patients with psoriasis. J Eur Acad Dermatol Venereol. 2016;30(2):282-287.

22. Kwon HH, Na SJ, Jo SJ, Youn JI. Epidemiology and clinical features of pediatric psoriasis in tertiary referral psoriasis clinic. $J$ Dermatol. 2012;39(3):260-264.
Clinical, Cosmetic and Investigational Dermatology

\section{Publish your work in this journal}

Clinical, Cosmetic and Investigational Dermatology is an international, peer-reviewed, open access, online journal that focuses on the latest clinical and experimental research in all aspects of skin disease and cosmetic interventions. This journal is included on PubMed. The manuscript management system is completely online

\section{Dovepress}

and includes a very quick and fair peer-review system, which is all easy to use. Visit http://www.dovepress.com/testimonials.php to read real quotes from published authors 